EPJ manuscript No.

(will be inserted by the editor)

\title{
Finite temperature dynamics of vortices in the two dimensional anisotropic Heisenberg model
}

\author{
Till Kamppeter ${ }^{1}$, Franz G. Mertens ${ }^{1}$, Angel Sánchez ${ }^{2}$, A. R. Bishop ${ }^{3}$, Francisco Domínguez-Adame ${ }^{4}$, and \\ N. Grønbech-Jensen ${ }^{3}$ \\ 1 Physikalisches Institut, Universität Bayreuth, D-95440 Bayreuth, Germany \\ 2 Grupo Interdisciplinar de Sistemas Complicados, Departamento de Matemáticas, Universidad Carlos III de Madrid, \\ E-28911 Leganés (Madrid), Spain \\ 3 Theoretical Division and Center for Nonlinear Studies, Los Alamos National Laboratory, New Mexico 87545, U.S.A. \\ 4 Grupo Interdisciplinar de Sistemas Complicados, Departamento de Física de Materiales, Facultad de Físicas, \\ Universidad Complutense, E-28040 Madrid, Spain \\ e-mail: till.kamppeter@theo.phy.uni-bayreuth.de, franz.mertens@theo.phy.uni-bayreuth.de, anxo@math.uc3m.es, \\ arb@idun.lanl.gov, adame@valbuena.fis.ucm.es, ngj@viking.lanl.gov
}

Received: February 1, 2008/ Revised version:

\begin{abstract}
We study the effects of finite temperature on the dynamics of non-planar vortices in the classical, two-dimensional anisotropic Heisenberg model with XY- or easy-plane symmetry. To this end, we analyze a generalized Landau-Lifshitz equation including additive white noise and Gilbert damping. Using a collective variable theory with no adjustable parameters we derive an equation of motion for the vortices with stochastic forces which are shown to represent white noise with an effective diffusion constant linearly dependent on temperature. We solve these stochastic equations of motion by means of a Green's function formalism and obtain the mean vortex trajectory and its variance. We find a non-standard time dependence for the variance of the components perpendicular to the driving force. We compare the analytical results with Langevin dynamics simulations and find a good agreement up to temperatures of the order of $25 \%$ of the Kosterlitz-Thouless transition temperature. Finally, we discuss the reasons why our approach is not appropriate for higher temperatures as well as the discreteness effects observed in the numerical simulations.
\end{abstract}

PACS. 05.40. $+\mathrm{j}$ Fluctuation phenomena, random processes, and Brownian motion - 75.10.Hk Classical spin models - 75.30.-m Intrinsic properties of magnetically ordered materials

\section{Introduction}

In the past two decades, solitons and other nonlinear coherent excitations have become a very generic and useful paradigm for intrinsically nonlinear phenomena in many different fields [1,2, 3]. These excitations are especially important in low dimensional systems, in terms of their relationship to key questions such as the existence of long range order, the mechanisms of phase transitions or the response to external influences [ [4]. Unfortunately, for most problems of interest or in applications, it is not possible to develop an exact theory of soliton dynamics or statistical mechanics, either because the corresponding equation of motion is not integrable or because perturbation terms added to it in order to account for relevant effects destroy integrability. As a consequence, much effort has been devoted to develop approximate techniques allowing one to gain insight into soliton behavior. Among those, a very useful procedure is that of collective variables or coordinates [5], which yields very accurate results for soliton-like objects with a well localized spatial structure. Collective coordinate techniques have, in addition, the advantages of their mathematical simplicity and their applicability to very many perturbed soliton-bearing equations, including most of those which are physically relevant. The validity of this kind of calculations has provided grounds to what is nowadays called the "particle-like picture" of solitons: In view of the fact that a global coordinate, such as their center of mass, is enough to describe their behavior under perturbations, it has been concluded that solitons can be treated as point-like particles in many situations.

One important context where the above ideas are relevant is that of two-dimensional (2D) magnets and their collective excitations such as vortices or domain walls. This is a far from an academic subject: Indeed, in the last few years several classes of materials have been found or fabricated for which magnetic interactions within planes of their crystalline structure are much stronger than between these planes, and therefore the magnetic properties are basically 2D. Materials in these classes include, for 
T. Kamppeter et al.: Dynamics of non-planar vortices ...

instance, layered magnets (such as $\mathrm{Rb}_{2} \mathrm{CrCl}_{4}$ ), graphite intercalated compounds (such as $\mathrm{CoCl}_{2}$ ), magnetic lipid layers (such as manganese stearate), and high $T_{c}$ superconductors (see references in, e. g., [6]). Many of these systems can be described by the classical 2D anisotropic Heisenberg model with XY- or easy-plane symmetry, given by

$$
H=-J \sum_{\langle m, n\rangle}\left[S_{x}^{m} S_{x}^{n}+S_{y}^{m} S_{y}^{n}+(1-\delta) S_{z}^{m} S_{z}^{n}\right],
$$

where the subindices $x, y$ or $z$ stand for the spin components, $0<\delta \leq 1$, and $\langle m, n\rangle$ labels nearest neighbors of a square lattice. Among its excitations, specially interesting ones are vortices, that are planar (i. e., with null $S_{z}$ components) if $\delta>0.297$ and non-planar (i. e., with localized $S_{z}$ structure) if $\delta<0.297$ [7. 8 . Such non-planar vortices are the specific object of our study as reported in the remainder of the paper; however, the ideas we will be discussing are general enough to be of interest in other, related contexts where the system behavior is governed by soliton-like structures.

The first application of a collective variable technique to the motion of magnetic vortices and other nonlinear magnetic excitations was carried out by Thiele [9, 10]. For steady state motion, when the shape $\mathbf{S}(\mathbf{r}, t)$ of the excitation in the continuum limit is rigid, he used the travelling wave ansatz $\mathbf{S}(\mathbf{r}, t)=\mathbf{S}(\mathbf{r}-\mathbf{X}(t))$ with constant velocity $\dot{\mathbf{X}}$ (the dot stands for derivative with respect to time) and derived the following equation of motion,

$$
\mathbf{G}_{V} \times \dot{\mathbf{X}}+\mathbf{F}=0
$$

where $\mathbf{F}$ is a static force, due to either an external field or the interactions with other excitations. The gyrovector $\mathbf{G}_{V}$, in turn, is an intrinsic quantity, produced by the excitation itself and depending on its specific type. $\mathbf{G}_{V}$ is perpendicular to the XY-plane; therefore, the gyrocoupling force $\mathbf{G}_{V} \times \dot{\mathbf{X}}$ is formally equivalent to the Lorentz force. Interestingly, Thiele's equation, Eq. (2), is first order, thus leading to non-Newtonian vortex dynamics. This is somewhat unusual, as in many cases solitons are found to behave as Newtonian point-like particles [5], obeying Newton's second law or its relativistic generalization. We return to this point below.

The next step beyond Thiele's approach was not taken until very recently, when Mertens et al. 11 developed a generalized collective variable theory for nonlinear coherent excitations in classical systems with arbitrary Hamiltonians. Previously, Wysin et al. 12 had tried to generalize Thiele's equation by allowing the vortex shape to depend on the vortex velocity. In this way they derived a second order (Newtonian) equation of motion, but it was found that it did not agree with the simulations [11, 13]. Therefore, in [1] it was proposed that the dynamics of a single excitation is governed by a hierarchy of equations of motion for the excitation center $\mathbf{X}(t)$. In addition, the Newtonian or non-Newtonian character of the equation of motion was clarified: It was found that the type of the excitation determines on which levels the hierarchy can be truncated consistently: So-called gyrotropic (with $\left|\mathbf{G}_{V}\right| \neq 0$ ) excitations are governed by odd-order equations and thus do not have Newtonian dynamics. Nongyrotropic excitations are described by even-order equations, i. e. by Newton's equation in the first approximation. This is the situation for, e. g., domain walls. The theory in [11] was applied to non-planar (gyrotropic) vortices of the $2 \mathrm{D}$ anisotropic Heisenberg model, and it was shown that their dynamics is fully captured by the third-order equation, fifth-order corrections being negligible [11.

Since the zero temperature dynamics of non-planar vortices is completely understood, in this paper we now concern ourselves with the study of non-planar vortex dynamics in the 2D Heisenberg ferromagnet at nonzero temperatures. The purpose of this research is twofold: From the theoretical point of view, it is important to learn whether and when the vortex motion description in terms of an effective particle dynamics holds, and what are its main characteristics. In addition, the non-Newtonian character of non-planar vortices could be modified by temperature, or the details of the dynamics could change as to eliminate the need to go beyond a first order equation. We note that if a collective coordinate theory at finite temperature could be worked out, it would provide a first step towards a statistical mechanics description of the model behavior in terms of a vortex gas 14, as in the case of one-dimensional soliton bearing systems [15. On the other hand, from the experimental point of view, insofar as the motion of vortices has measurable consequences in inelastic neutron scattering [16] and nuclear magnetic resonance experiments [17, the effects of finite temperature on vortex dynamics can have signatures in those measurements. The study we carry out here is then necessary if there is hope to compare the theoretical results to actual experiments.

The presentation of our results proceeds as follows: Section 2 contains the study of the free and the damped vortex dynamics and the derivation of the corresponding collective coordinate theory. At this point the study is still deterministic, i. e., at zero temperature. Section 3 discusses how we incorporate the Langevin noise term to the equations of motion. Afterwards, the collective coordinate calculation is extended to the resulting Langevin-LandauLifshitz equation, and the mean vortex trajectory and its variance are computed. Section 4 contains a thorough discussion of the comparison of the theory to the numerical Langevin dynamics simulation and the discussion of the main features of the vortex motion. Finally, Section 5 is devoted to the summary of our main conclusions.

\section{Zero temperature dynamics}

Our starting point is the damped Landau-Lifshitz equation, which reads

$$
\frac{\mathrm{d} \mathbf{S}^{m}}{\mathrm{~d} t}=-\mathbf{S}^{m} \times \frac{\partial H}{\partial \mathbf{S}^{m}}-\epsilon \mathbf{S}^{m} \times \frac{\mathrm{d} \mathbf{S}^{m}}{\mathrm{~d} t},
$$

where $\mathbf{S}^{m}$ is the spin vector at lattice site $m, H$ is the Hamiltonian, in our case that of the anisotropic Heisen- 
berg model (11), and $\epsilon$ is the damping parameter. Following Refs. [9], 10] and [18] we have chosen Gilbert damping [19], chiefly because it is isotropic, meaning that all the spin components are equally damped, in contrast to the Landau-Lifshitz damping 20]. As stated in the Introduction, our approach to the problem of vortex dynamics will be both analytical and numerical: We first derive equations of motion for the vortex center $\mathbf{X}(t)$, and afterwards we compare with numerical simulations for our model, i. e., with results from numerical integration of (3) including noise (see Section 31). The study of the deterministic (i.e., zero temperature) case we present in this section is an obviously necessary first step in order to be able to understand later the problem for the full Langevin equation.

To proceed, following [11 we assume that the shape of a collective excitation depends on the velocity $\dot{\mathbf{X}}$ and, as shown in [11], in general also on higher order derivatives of $\mathbf{X}(t)$. The corresponding generalized travelling wave Ansatz is

$$
\mathbf{S}(\mathbf{r}, t)=\mathbf{S}\left(\mathbf{r}-\mathbf{X}, \dot{\mathbf{X}}, \ddot{\mathbf{X}}, \ldots, \mathbf{X}^{(n)}\right),
$$

which yields an $(n+1)$-th order differential equation for $\mathbf{X}(t)$. As mentioned above, for gyrotropic excitations only odd-order equations are relevant, and, in the case of the non-planar vortices, it turned out that the third-order equation is sufficient to describe accurately all simulations without damping [11]. Therefore, in this paper we use the Ansatz (田) with $n=2$ and apply it to the general case, i.e., in the presence of damping. Instead of using the Hamiltonian procedure described in [11], we will obtain the collective variable equations of motion in a much more direct way by performing the following operations with (3): leaving out damping for the moment, we calculate

$$
\begin{aligned}
& \mathbf{S}\left(\frac{\partial \mathbf{S}}{\partial X_{i}} \times \frac{\mathrm{d} \mathbf{S}}{\mathrm{d} t}\right)=-\mathbf{S}\left(\frac{\partial \mathbf{S}}{\partial X_{i}} \times\left[\mathbf{S} \times \frac{\delta H}{\delta \mathbf{S}}\right]\right)= \\
&=-S^{2} \frac{\delta H}{\delta \mathbf{S}} \frac{\partial \mathbf{S}}{\partial X_{i}}=-S^{2} \frac{\partial \mathcal{H}}{\partial X_{i}}
\end{aligned}
$$

with $i=1,2$ in the case of our 2D system. $\mathcal{H}$ is the Hamiltonian density. According to our ansatz we insert on the l.h.s.

$$
\frac{\mathrm{d} \mathbf{S}}{\mathrm{d} t}=\frac{\partial \mathbf{S}}{\partial X_{j}} \dot{X}_{j}+\frac{\partial \mathbf{S}}{\partial \dot{X}_{j}} \ddot{X}_{j}+\frac{\partial \mathbf{S}}{\partial \ddot{X}_{j}} \dddot{X}_{j},
$$

integrate over $\mathbf{r}$ and divide by $S^{2}$. In this way we obtain the same third-order equation as that obtained in Ref. [11], which used Hamilton equations:

$$
\mathbf{A} \dddot{\mathbf{X}}+\mathbf{M} \ddot{\mathbf{X}}+\mathbf{G} \dot{\mathbf{X}}=\mathbf{F}
$$

with force $\mathbf{F}$ given by

$$
F_{i}=-\int d^{2} r \frac{\partial \mathcal{H}}{\partial X_{i}}
$$

gyrotensor $\mathbf{G}$ expressed as

$$
\begin{array}{rl}
G_{i j}=S^{-2} \int d^{2} r & \mathbf{S} \frac{\partial \mathbf{S}}{\partial X_{i}} \times \frac{\partial \mathbf{S}}{\partial X_{j}}= \\
=\int d^{2} r & \left\{\frac{\partial \phi}{\partial X_{i}} \frac{\partial \psi}{\partial X_{j}}-\frac{\partial \phi}{\partial X_{j}} \frac{\partial \psi}{\partial X_{i}}\right\},
\end{array}
$$

mass tensor $\mathbf{M}$ with components

$$
\begin{aligned}
& M_{i j}=S^{-2} \int d^{2} r \mathbf{S} \frac{\partial \mathbf{S}}{\partial X_{i}} \times \frac{\partial \mathbf{S}}{\partial \dot{X}_{j}}= \\
&=\int d^{2} r\left\{\frac{\partial \phi}{\partial X_{i}} \frac{\partial \psi}{\partial \dot{X}_{j}}-\frac{\partial \phi}{\partial \dot{X}_{j}} \frac{\partial \psi}{\partial X_{i}}\right\},
\end{aligned}
$$

and third-order gyrotensor $\mathbf{A}$ given by

$$
\begin{aligned}
A_{i j}=S^{-2} \int d^{2} r \mathbf{S} \frac{\partial \mathbf{S}}{\partial X_{i}} \times \frac{\partial \mathbf{S}}{\partial \ddot{X}_{j}}= \\
=\int d^{2} r\left\{\frac{\partial \phi}{\partial X_{i}} \frac{\partial \psi}{\partial \ddot{X}_{j}}-\frac{\partial \phi}{\partial \ddot{X}_{j}} \frac{\partial \psi}{\partial X_{i}}\right\} .
\end{aligned}
$$

The classical spin is constrained to have a fixed magnitude which we set to unity. Therefore, we will evaluate below the expressions on the right hand sides using canonical fields $\phi=\arctan \left(S_{y} / S_{x}\right)$ and $\psi=S_{z}$ for the spin vector:

$$
\mathbf{S}=\sqrt{1-\psi^{2}} \cos \phi \mathbf{e}_{x}+\sqrt{1-\psi^{2}} \sin \phi \mathbf{e}_{y}+\psi \mathbf{e}_{z} .
$$

At this time, we move on to consider the Gilbert damping term in (3). The same operation sequence as above yields:

$$
\begin{aligned}
& \epsilon \mathbf{S}\left[\frac{\partial \mathbf{S}}{\partial X_{i}} \times\left(\mathbf{S} \times \frac{\mathrm{d} \mathbf{S}}{\mathrm{d} t}\right)\right]=\epsilon S^{2} \frac{\partial \mathbf{S}}{\partial X_{i}} \frac{\mathrm{d} \mathbf{S}}{\mathrm{d} t}= \\
& =\epsilon S^{2}\left[\frac{\partial \mathbf{S}}{\partial X_{i}} \frac{\partial \mathbf{S}}{\partial X_{j}} \dot{X}_{j}+\frac{\partial \mathbf{S}}{\partial X_{i}} \frac{\partial \mathbf{S}}{\partial \dot{X}_{j}} \ddot{X}_{j}+\frac{\partial \mathbf{S}}{\partial X_{i}} \frac{\partial \mathbf{S}}{\partial \ddot{X}_{j}} \dddot{X}_{j}\right]
\end{aligned}
$$

An integration over $\mathbf{r}$ gives three terms which can be combined with the three terms on the l.h.s. of (7), i. e., the damping appears in every order

$$
\begin{aligned}
(\mathbf{A}+\mathbf{a}) \dddot{\mathbf{X}}+(\mathbf{M}+\mathbf{m}) \ddot{\mathbf{X}}+(\mathbf{G}+\mathbf{g}) \dot{\mathbf{X}}= \\
=\hat{\mathbf{A}} \dddot{\mathbf{X}}+\hat{\mathbf{M}} \ddot{\mathbf{X}}+\hat{\mathbf{G}} \dot{\mathbf{X}}=\mathbf{F} .
\end{aligned}
$$

The components of the damping contribution to the tensors are

$$
\begin{gathered}
g_{i j}=\epsilon \int d^{2} r \frac{\partial \mathbf{S}}{\partial X_{i}} \frac{\partial \mathbf{S}}{\partial X_{j}}= \\
=\epsilon \int d^{2} r\left\{\left(1-\psi^{2}\right) \frac{\partial \phi}{\partial X_{i}} \frac{\partial \phi}{\partial X_{j}}+\frac{1}{1-\psi^{2}} \frac{\partial \psi}{\partial X_{j}} \frac{\partial \psi}{\partial X_{i}}\right\}
\end{gathered}
$$

$$
\begin{gathered}
m_{i j}=\epsilon \int d^{2} r \frac{\partial \mathbf{S}}{\partial X_{i}} \frac{\partial \mathbf{S}}{\partial \dot{X}_{j}}= \\
=\epsilon \int d^{2} r\left\{\left(1-\psi^{2}\right) \frac{\partial \phi}{\partial X_{i}} \frac{\partial \phi}{\partial \dot{X}_{j}}+\frac{1}{1-\psi^{2}} \frac{\partial \psi}{\partial \dot{X}_{j}} \frac{\partial \psi}{\partial X_{i}}\right\}
\end{gathered}
$$

$$
\begin{gathered}
a_{i j}=\epsilon \int d^{2} r \frac{\partial \mathbf{S}}{\partial X_{i}} \frac{\partial \mathbf{S}}{\partial \ddot{X}_{j}}= \\
=\epsilon \int d^{2} r\left\{\left(1-\psi^{2}\right) \frac{\partial \phi}{\partial X_{i}} \frac{\partial \phi}{\partial \ddot{X}_{j}}+\frac{1}{1-\psi^{2}} \frac{\partial \psi}{\partial \ddot{X}_{j}} \frac{\partial \psi}{\partial X_{i}}\right\}
\end{gathered}
$$


We note that the first-order part of (14) was already derived by Thiele 9].

Now, we address the problem of the explicit calculation of all the tensor components. This is possible only if the dynamic structure of the collective excitation is known. The Hamiltonian density derived from (1) reads [7]

$$
\begin{aligned}
\mathcal{H}=\frac{J S^{2}}{2}\left\{\left(1-\psi^{2}\right)(\nabla \phi)^{2}+\right. & \delta\left[4 \psi^{2}-(\nabla \psi)^{2}\right]+ \\
& \left.+\frac{1}{1-\psi^{2}}(\nabla \psi)^{2}\right\} .
\end{aligned}
$$

In 11] the Hamilton equations were considered for a nonplanar vortex in the center of a circular system with free boundary conditions. The vortex structure is complicated in an inner region $0 \leq r \leq a_{c} \approx 3 r_{\mathrm{v}}$, where

$$
r_{\mathrm{v}}=\frac{1}{2} \sqrt{\frac{1-\delta}{\delta}}
$$

characterizes the vortex core [7]. $\delta$ is the anisotropy parameter in (11). Recalling that non-planar vortices are stable for $0<\delta<0.297$ for a square lattice. we will use $\delta=0.1$ for our simulations. We note that the inner region contributes very little to the integrals in (10), (11) and (15)-(17); except for (9), the dominant contributions stem from the outer region $a_{c} \leq r \leq L$, if we choose a large system radius $L$. Here the vortex has the following dynamic structure, which is known to be a very accurate description from simulations [11]:

$$
\phi=\phi_{0}+\phi_{1}+\phi_{2}, \quad \psi=\psi_{0}+\psi_{1}+\psi_{2}
$$

with

$$
\begin{gathered}
\phi_{0}=q \tan ^{-1} \frac{x_{2}}{x_{1}}, \\
\phi_{1}=p\left(x_{1} \dot{X}_{1}+x_{2} \dot{X}_{2}\right), \\
\phi_{2}=\frac{q}{8 \delta} \ln \frac{r}{e L}\left(x_{2} \ddot{X}_{1}-x_{1} \ddot{X}_{2}\right), \\
\psi_{0} \sim p \sqrt{\frac{r_{\mathrm{v}}}{r}} \exp \left(-r / r_{\mathrm{v}}\right), \\
\psi_{1}=\frac{q}{4 \delta r^{2}}\left(x_{2} \dot{X}_{1}-x_{1} \dot{X}_{2}\right),
\end{gathered}
$$

and

$$
\psi_{2}=\frac{p}{4 \delta}\left(x_{1} \ddot{X}_{1}+x_{2} \ddot{X}_{2}\right) .
$$

Here $q= \pm 1$ is the vorticity and $p= \pm 1$ is the polarization, which determines to which side the out-of-plane structure of the vortex points. Straightforward integrations then yield the expressions of the tensor components:

$$
G_{i j}=G \epsilon_{i j}, \quad G=2 \pi p q,
$$

$$
\begin{gathered}
M_{i j}=M \delta_{i j}, \quad M=\frac{\pi q^{2}}{4 \delta} \ln \frac{L}{a_{c}}+C_{M}, \\
A_{i j}=A \epsilon_{i j}, \quad A=\frac{G}{16 \delta}\left(L^{2}-a_{c}^{2}\right)+C_{A}, \\
g_{i j}=g \delta_{i j}, \quad g=\epsilon \pi q^{2} \ln \frac{L}{a_{c}}+C_{g}, \\
m_{i j}=m \epsilon_{i j}, \quad m=\epsilon \frac{G}{4}\left(L^{2}-a_{c}^{2}\right)+C_{m},
\end{gathered}
$$

and

$$
\begin{array}{r}
a_{i j}=a \delta_{i j}, \quad a=\epsilon \frac{\pi q^{2}}{8 \delta}\left\{\frac{1}{2}\left(L^{2} \ln L-a_{c}^{2} \ln a_{c}\right)-\right. \\
\left.\frac{1}{4}\left(L^{2}-a_{c}^{2}\right)\right\}+C_{a},
\end{array}
$$

where $\delta_{i j}$ is the $2 \mathrm{D}$ unit matrix, $\epsilon_{i j}$ is the antisymmetric tensor, and the different constants $C$ are the contributions from the inner region of the vortex. We see that in every odd-order of (14) a symmetric damping matrix is combined with an antisymmetric normal (non-damping) matrix, and vice versa for the even orders. Moreover the size dependence of the $n$-th order damping components is the same as that of the $(n+1)$-th order normal components. The first-order damping elements 30 were already evaluated in [6] and [10].

For the solution of the equation of motion (14) we proceed as in Ref. [11]: We consider small displacements $\mathbf{x}$ from a mean trajectory $\mathbf{X}^{0}$, on which the vortex is driven by $\mathbf{F}$

$$
\mathbf{X}(t)=\mathbf{X}^{0}(t)+\mathbf{x}(t)
$$

We will denote the components of $\mathbf{x}$ by $x_{1}$ and $x_{2}$, with the caveat that they should not be confused with the components of $\mathbf{r}$ in Eqs. (21) through (26). In view of our simulations, we consider the situation where the force is always pointing in the $X_{1}$-direction and expand to firstorder around $X_{1}(0)=R_{0}$ (this is justified because in our simulations $F_{0}$, and even more $F_{0}^{\prime}$, is very small), i. e.,

$$
F=F_{0}+F_{0}^{\prime} x_{1}
$$

For $X_{i}^{0}(t)$ we obtain two coupled linear third-order equations. Taking initial conditions $X_{1}^{0}(0)=R_{0}, X_{2}^{0}(0)=0$ the solutions are

$$
\begin{aligned}
& X_{1}^{0}=R_{0}+\frac{F_{0}}{F_{0}^{\prime}}[\exp (t / \tau)-1], \\
& X_{2}^{0}=\frac{G}{g} \frac{F_{0}}{F_{0}^{\prime}}[\exp (t / \tau)-1],
\end{aligned}
$$

where $\tau$ is determined by a cubic equation. The mean trajectory is a straight line $X_{2}^{0}=G / g\left(X_{1}^{0}-R_{0}\right)$, which 
slightly deviates from the $X_{2}$-axis. The angle $g / G$ is small because $g \sim \epsilon$, where we choose small damping constants $\epsilon$ in the simulations. As $\tau$ is of the order of $G^{2} /\left(g F_{0}^{\prime}\right)$ it is very large, in fact much larger than our integration times. Therefore one can expand (35) and one get a constant velocity on the mean trajectory: $\dot{X}_{1}^{0}=g F_{0} / G^{2}, \dot{X}_{2}^{0}=$ $F_{0} / G$.

The motion around the mean trajectory is obtained by solving the two coupled linear third-order equations for the displacements $\mathbf{x}(t)$ using the Ansatz

$$
x_{i}=x_{i}^{0} \exp [-(\beta-i \omega) t] .
$$

We find

$$
\begin{aligned}
\beta-i \omega= & \frac{ \pm i M+m}{2(A \pm i a)} \pm \\
& \pm \frac{\sqrt{( \pm i M+m)^{2}-4(A \pm i a)(G \pm i g)}}{2(A \pm i a)}
\end{aligned}
$$

with amplitude ratios $\kappa=x_{2}^{0} / x_{1}^{0}= \pm 1$ and phase differences $\pm \pi / 2$, where we have set $F_{0}^{\prime}=0$ for simplicity. With $F_{0}^{\prime} \neq 0$ Eq. (38) becomes even more complicated and $|\kappa| \neq 1$. The separation of real and imaginary parts leads to cumbersome formulas. Therefore we compute the frequencies $\omega_{1,2}$ and the relaxation constants $\beta_{1,2}$ as a function of the parameters $\epsilon$ and $L$; we choose $q=p=1$ for the charges and $\delta=0.1$ for the anisotropy. The $a_{c}$-dependent parts in (28)-(31) can be combined with the constants $C_{M}$ etc.; the combined constants can be neglected for large systems. As for the frequencies, $\omega_{1,2}$ turn out to be very close to each other; hence, the important parameters will instead be their mean and difference. Examples of their numerical values for a system of radius $L=24$ are

$$
\omega_{c}=\sqrt{\omega_{1} \omega_{2}} \approx 0.05, \quad \Delta \omega=\omega_{2}-\omega_{1} \approx 0.01,
$$

for a wide range of damping values (up to $\epsilon=0.05$ ), whereas for fixed $\epsilon$ the frequencies decrease with $1 / L$ up to rather large systems $(L=5000)$. Plots of $\omega_{c}$ and $\Delta \omega$ as a function of $\epsilon$ and $L$ can be found in 21] (note, however that the caption under Fig. 1 of 21 must read 48 instead of 24$)$.

In the simulations the purpose of the damping is to dissipate the energy which is supplied to the system by the noise. Therefore we must know the range of $\epsilon$ (for a given system size) in which the frequencies are not influenced by the damping. As shown in 21 , this range is defined by the condition

$$
\epsilon L \ll 5
$$

The relaxation constants $\beta_{1,2}$ are nearly equal and the mean value is $\beta_{c}=\epsilon / 8$, and for the above range $\omega_{c}$ and $\Delta \omega$ are related to the parameters $G, M$, and $A$ in a very simple way 11

$$
\omega_{c}=\sqrt{\frac{G}{A}} \sim \frac{1}{L}, \quad \Delta \omega=\frac{M}{A} \sim \frac{\ln L}{L^{2}} .
$$

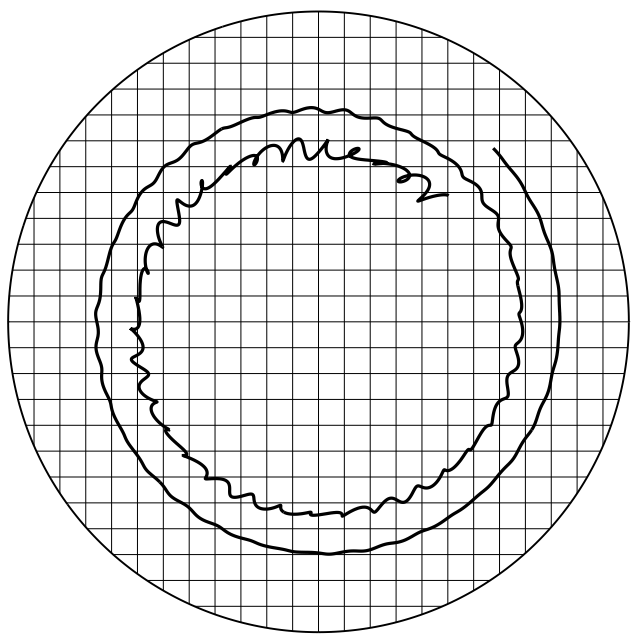

Fig. 1. Sketch of the vortex motion as governed by the LandauLifshitz equation with Gilbert damping. The plot is approximate and does not correspond to an actual simulation.

Finally we briefly discuss the shape of the trajectories. We first consider the motion in a frame which is moving along the mean trajectory $\mathbf{X}^{0}(t)$ : The general solutions for the displacements $x_{i}(t)$ are linear superpositions of (37) with $\omega_{1,2}$. Both $x_{i}(t)$ exhibit a very pronounced beat because $\omega_{1,2}$ are nearly equal. The orbits $x_{1}\left(x_{2}\right)$ are Lissajous curves, which can look very intricate for certain parameter ranges. We go into the laboratory frame by adding $\mathbf{X}^{0}(t)$. Without the splitting of $\omega_{1,2}$ we would get a cycloid. Due to the splitting we finally get a superposition of two cycloids, which are damped because of $\beta_{1,2}$. A cartoon of the vortex motion on a circular system is sketched in Fig. 11. Here the vortex is driven by an image force which points in radial direction (see Section 4 ). Without damping, the mean trajectory $\mathbf{X}^{0}(t)$ would be a circle, due to the gyrocoupling force. With damping, the circle converts to an outward spiral. However, the radial motion is very exaggerated in the sketch, and the same is true of the damping of the cycloidal oscillations around the mean trajectory. The amplitude of these oscillations in fact remains of the order of a lattice constant for a long time.

\section{Finite temperature dynamics}

\subsection{Derivation of the vortex equation of motion}

In order to study the finite temperature dynamics of vortices, we must introduce thermal noise in the LandauLifshitz equation with damping, Eq. (3). However, we cannot simply add independent noise terms to these three equations, because if we do so we do not arrive to something in the form of Langevin equations (all components of $d \mathbf{S} / d t$ appear in each equation due to the cross-product). Therefore we must first take all $d \mathbf{S} / d t$-terms to the l.h.s., casting it explicitly into a first order equation, and only 
then introduce the three white-noise terms $\eta_{\alpha}^{\prime}(\mathbf{r}, t)$, yielding:

$$
\begin{aligned}
\frac{\mathrm{d} \mathbf{S}}{\mathrm{d} t}=\frac{1}{1+\epsilon^{2} S^{2}}\{-\mathbf{S} \times & \frac{\delta H}{\delta \mathbf{S}}+ \\
& \left.+\epsilon \mathbf{S} \times\left[\mathbf{S} \times \frac{\delta H}{\delta \mathbf{S}}\right]\right\}+\boldsymbol{\eta}^{\prime}
\end{aligned}
$$

with

$$
\begin{aligned}
\left\langle\eta_{\alpha}^{\prime}(\mathbf{r}, t)\right\rangle & =0 \\
\left\langle\eta_{\alpha}^{\prime}(\mathbf{r}, t) \eta_{\beta}^{\prime}\left(\mathbf{r}^{\prime}, t^{\prime}\right)\right\rangle & =2 \epsilon k_{\mathrm{B}} T \delta\left(\mathbf{r}^{\prime}-\mathbf{r}\right) \delta\left(t^{\prime}-t\right) \delta_{\alpha \beta},
\end{aligned}
$$

where $\alpha, \beta=1,2,3$. Now we take $\boldsymbol{\eta}^{\prime}$ to the l.h.s. and undo the above procedure, i. e., we write (42) in the same form as (3), but with $\dot{\mathbf{S}}-\boldsymbol{\eta}^{\prime}$ instead of $\dot{\mathbf{S}}$. We thus arrive at

$$
\frac{\mathrm{d} \mathbf{S}}{\mathrm{d} t}=-\mathbf{S} \times \frac{\delta H}{\delta \mathbf{S}}-\epsilon \mathbf{S} \times \frac{\mathrm{d} \mathbf{S}}{\mathrm{d} t}+\boldsymbol{\eta}
$$

with

$$
\boldsymbol{\eta}=\boldsymbol{\eta}^{\prime}-\epsilon\left(\mathbf{S} \times \boldsymbol{\eta}^{\prime}\right)
$$

If we now compute the variances of $\boldsymbol{\eta}$, we find that the width of the distribution for the component parallel to the spin vector is $\sigma_{0}=\sqrt{2 \epsilon k_{\mathrm{B}} T}$, while the widths for the perpendicular components are $\sigma_{0} \sqrt{1+\epsilon^{2} S^{2}}$. In the Langevin dynamics simulation we will apply the constraint $|\mathbf{S}|=1$, which means here that only the perpendicular components are relevant. Thus we can replace $\boldsymbol{\eta}$ in (45) by $\boldsymbol{\eta}^{\prime}$ if we correct the widths by a factor of $\sqrt{1+\epsilon^{2}}$. Taking into account that in our simulations we will be using values of $\epsilon$ of the order of $10^{-3}$ (see Sec. 郋), we will neglect the correction factor in the following.

As in the previous section, we calculate

$$
\mathbf{S}\left(\frac{\partial \mathbf{S}}{\partial X_{i}} \times \boldsymbol{\eta}\right)=\left(\mathbf{S} \times \frac{\partial \mathbf{S}}{\partial X_{i}}\right) \boldsymbol{\eta},
$$

integrate over $\mathbf{r}$ and combine this with the results of the previous section; we have thus found the collective coordinate equation in the presence of noise, namely

$$
\hat{\mathbf{A}} \dddot{\mathbf{X}}+\hat{\mathbf{M}} \ddot{\mathbf{X}}+\hat{\mathbf{G}} \dot{\mathbf{X}}=\mathbf{F}+\mathbf{F}^{\mathrm{st}},
$$

where the stochastic force is given by

$$
F_{i}^{\mathrm{st}}=\frac{1}{S^{2}} \int \mathrm{d}^{2} r\left(\mathbf{S} \times \frac{\partial \mathbf{S}}{\partial X_{i}}\right) \boldsymbol{\eta}(\mathbf{r}, t) .
$$

To achieve a complete understanding of the vortex dynamics as described by Eq. (48), we need to know the mean $\left\langle F_{i}^{\text {st }}\right\rangle$ and the variance $\operatorname{Var}\left(F_{i}^{\text {st }}\right)$. We define

$$
F_{i}^{\mathrm{st}}=\int \mathrm{d}^{2} r f_{i}^{(\alpha)} \eta_{\alpha}, \quad f_{i}^{(\alpha)}=\frac{1}{S^{2}} \epsilon_{\alpha \beta \gamma} S_{\beta} \frac{\partial S_{\gamma}}{\partial X_{i}}
$$

where summation over repeated indices is implicitly understood. The mean is easily shown to be zero, whereas for the correlation functions [22], from Eq. (44) we obtain

$$
\begin{aligned}
& \left\langle F_{i}^{\mathrm{st}}(t) F_{i}^{\mathrm{st}}\left(t^{\prime}\right)\right\rangle= \\
& \quad=2 \epsilon k_{\mathrm{B}} T \delta\left(t-t^{\prime}\right) \int \mathrm{d}^{2} r f_{i}^{(\alpha)}(\mathbf{r}) f_{i}^{(\alpha)}(\mathbf{r}) .
\end{aligned}
$$

Instead of the $S_{\alpha}$ we introduce the fields $\phi$ and $\psi$ in (12) and thereby fulfill the constraint $|\mathbf{S}|=1$. After some algebra we obtain

$$
\begin{array}{r}
\operatorname{Var}\left(F_{i}^{\mathrm{st}}\right)=2 \epsilon k_{\mathrm{B}} T \int d^{2} r\left\{\left(1-\psi^{2}\right)\left(\frac{\partial \phi}{\partial X_{i}}\right)^{2}+\right. \\
\left.\frac{1}{1-\psi^{2}}\left(\frac{\partial \psi}{\partial X_{i}}\right)^{2}\right\} .
\end{array}
$$

We note that in this equation the leading contribution comes from the static vortex structure, as given by Eqs. (21) and (24):

$$
\begin{array}{r}
\operatorname{Var}\left(F_{i}^{\mathrm{st}}\right)=2 \pi \epsilon k_{\mathrm{B}} T \int_{0}^{L} d r r\left\{\frac{1-\psi_{0}(r)^{2}}{r^{2}}+\right. \\
\left.\frac{\left(\psi_{0}^{\prime}(r)\right)^{2}}{1-\psi_{0}(r)^{2}}\right\} .
\end{array}
$$

As $\psi_{0}$ decays exponentially, the second integral is independent of $L$, while the first one grows logarithmically. This suggests that, in order to approximately calculate $\operatorname{Var}\left(F_{i}^{\text {st }}\right)$, we can divide the integral in an outer part from $a_{c} \leq r \leq L$ and a core part $C\left(a_{c}\right)$. By doing so we can write

$$
\operatorname{Var}\left(F_{i}^{\mathrm{st}}\right)=2 \epsilon k_{\mathrm{B}} T \cdot \pi\left\{\ln \frac{L}{a_{c}}+C\left(a_{c}\right)\right\}
$$

which implies that the stochastic forces can be represented as white noise on the level of the collective coordinates with the properties $\left\langle F_{i}^{\text {st }}\right\rangle=0$ and

$$
\left\langle F_{i}^{\mathrm{st}}(t) F_{j}^{\mathrm{st}}\left(t^{\prime}\right)\right\rangle=D_{V} \delta_{i j} \delta\left(t-t^{\prime}\right),
$$

where the effective vortex diffusion constant $D_{V}$ is determined by the r.h.s. of (54). We recall that the diffusion constant $D$ on the microscopic level, i. e., the one we will use in the simulations, is $D=2 \epsilon k_{\mathrm{B}} T$.

The core contribution $C\left(a_{c}\right)$ in the integral (53) cannot be calculated accurately by using $\psi_{0}(r)$ from the continuum limit [7]. Therefore, we have computed the full integral (53) using for $\psi_{0}$ an ad-hoc function which was fitted to the static vortex structure as obtained from the simulations at zero temperature [21,24. The results for $L=24$ and $\delta=0.03,0.10$, and 0.30 are $D_{V} / D=10.02,12.08$, and 14.18 , respectively. 
T. Kamppeter et al.: Dynamics of non-planar vortices ...

\subsection{Solution of the equation of motion}

We now turn to the solution of Eq. (48). As the force $\mathbf{F}$, which drives the vortex, can be expanded up to first order around the mean trajectory as discussed in Sec. 2, we find the linear equation

$$
\hat{\mathbf{A}} \dddot{\mathbf{x}}+\hat{\mathbf{M}} \ddot{\mathbf{x}}+\hat{\mathbf{G}} \dot{\mathbf{x}}-\mathbf{f} \mathbf{x}=\mathbf{F}^{\mathrm{st}}
$$

with

$$
\mathbf{f}=\left(\begin{array}{cc}
F_{0}^{\prime} & 0 \\
0 & 0
\end{array}\right)
$$

Thus we can use the Green's function formalism to obtain a formal solution. This proceeds in two steps. First, the Green's function matrix is obtained from the solution to the equations

$$
\hat{\mathbf{A}} \dddot{\mathbf{g}}_{i}+\hat{\mathbf{M}} \ddot{\mathbf{g}}_{i}+\hat{\mathbf{G}} \dot{\mathbf{g}}_{i}-\mathbf{f g}_{i}=\delta(t) \mathbf{I}_{i}
$$

where $\mathbf{g}_{i}$, with $i=1,2$, are the two columns of the Green's function matrix, $\mathbf{I}_{i}$ are the corresponding columns of the identity matrix, and suitable conditions have to be imposed on both eqs. (58). Once the Green's matrix $\mathbf{G}$ has been calculated, the second step is to solve the stochastic problem (56). Its solution is then exactly given by

$$
\mathbf{x}(t)=\mathbf{x}_{h}(t)+\int_{0}^{t} d s \mathbf{G}(t-s) \mathbf{F}^{\mathrm{st}}(s),
$$

where $\mathbf{x}_{h}(t)$ stands for the solution of the homogeneous version of Eq. (56). We note that $\mathbf{G}$ should not be confused with the gyrocoupling tensor in Eqs. (7), (9), and (27).

Let us now discuss the first part of the calculation, i.e., the computation of the Green's matrix G. Eqs. (58) above need to be suplemented with the following conditions: $\mathbf{G}$, $\dot{\mathbf{G}}$, and $\ddot{\mathbf{G}}$ vanish for $t \leq 0$, and

$$
\begin{aligned}
& \mathbf{G}\left(0^{+}\right)=\dot{\mathbf{G}}\left(0^{+}\right)=\left(\begin{array}{ll}
0 & 0 \\
0 & 0
\end{array}\right), \\
& \ddot{\mathbf{G}}\left(0^{+}\right)=\hat{\mathbf{A}}^{-1}=\frac{1}{a^{2}+A^{2}}\left(\begin{array}{cc}
a-A \\
A & a
\end{array}\right) .
\end{aligned}
$$

In order to find the columns $\mathbf{g}_{i}$ of the Green's matrix, we take the Ansatz

$$
\mathbf{g}_{i}(t)=\sum_{k=1}^{6} c_{k}^{(i)}\left(\begin{array}{l}
a_{k} \\
b_{k}
\end{array}\right) \exp \left(\lambda_{k} t\right) \theta(t),
$$

where $\theta(t)$ is the Heaviside function, $a_{k}$ and $b_{k}$ form the eigenvectors belonging to the eigenvalues $\lambda_{k}$ of the homogeneous problem, i.e., Eq. (58) with its r.h.s. set to zero, and $c_{k}^{(i)}$ are the unknown amplitudes in the linear combination. The eigenvalues are already known from the previous section: one is zero, one is $1 / \tau$ [see below Eq. (36)], and the other four are given by Eq. (38). All that remains is to insert the Ansatz (62) in Eq. (58) and find the values for $c_{k}^{(i)}$ from the corresponding system of algebraic equations. Their expression is rather cumbersome and therefore we do not present it here insofar as the derivation is straightforward.

Once the $c_{k}^{(i)}$ and hence $\mathbf{G}$ are known, we can move to the second part of the procedure, namely to find the trajectory and to evaluate its relevant moments. It is evident from Eq. (59) that the mean trajectory will be exactly the same as that of the deterministic case, because the average of the integral of $\mathbf{F}^{s t}$ vanishes. We will therefore concentrate on the variances,

$$
\sigma_{i j}^{2}(t)=\left\langle x_{i} x_{j}\right\rangle-\left\langle x_{i}\right\rangle\left\langle x_{j}\right\rangle
$$

Using the expression (59) it can immediately be seen that

$$
\sigma_{i j}^{2}(t)=\sum_{k=1}^{2} \int_{0}^{t} d t^{\prime} D_{V} G_{i k}\left(t-t^{\prime}\right) G_{j k}\left(t-t^{\prime}\right),
$$

where $G_{i j}$ stand for the elements of the Green's matrix. Once again, the calculation is simple but tedious, due to the many terms involved by the product of the Green's matrix elements. Aside from this, the expression is easily obtained as the integrals involve only exponentials. As an example, we present a summary of the calculation of $\sigma_{11}^{2}$, which is the simplest element of the variance matrix. Nevertheless, in order to facilitate the presentation and the subsequent discussion we have made the following simplifications: i) $\omega_{1}=\omega_{2}=\omega_{c}$ and $\beta_{1}=\beta_{2}=\beta_{c}$, because the splittings are very small [see Eq. (41) and above]; ii) $\omega_{c}^{2}+\beta_{c}^{2} \simeq \omega_{c}^{2}$ because $\beta_{c}=\epsilon / 8$ and $\epsilon=0.002$ in the simulations, implying $\beta_{c}$ is two orders of magnitude smaller than $\omega_{c}$ as given by Eq. (39); iii) $A^{2}+a^{2} \simeq A^{2}$, because $a / A=\mathrm{O}(\epsilon)$, see Eqs. (29) and (32), and iv) exponential terms involving $t / \tau$ are expanded to first order, because $\tau$ is much larger than our integration times; see below Eq. (36). Within these approximations, it can be shown that

$$
\begin{aligned}
\sigma_{11}^{2}(t)= & \frac{D_{V}}{A^{2} \omega_{c}^{4}}\left[t+\frac{1}{4 \beta_{c}}\left(1-e^{-2 \beta_{c} t}\right)-\right. \\
& \left.-\frac{2}{\omega_{c}} e^{-\beta_{c} t} \sin \omega_{c} t+\frac{1}{4 \omega_{c}} e^{-2 \beta_{c} t} \sin 2 \omega_{c} t\right] .
\end{aligned}
$$

For small times, $t \ll 1 / \beta_{c}$, we are left with an expression which implies linear behavior plus oscillations, given by

$$
\sigma_{11}^{2}(t)=\frac{D_{V} t}{A^{2} \omega_{c}^{4}}\left(\frac{3}{2}-2 \frac{\sin \omega_{c} t}{\omega_{c} t}+\frac{\sin 2 \omega_{c} t}{4 \omega_{c} t}\right) .
$$

We note that this function increases monotonously and that it has no extrema but only inflection points. For large times, $t \gg 1 / \beta_{c}$, only the first term of Eq. (65) remains, and the variance becomes a straight line,

$$
\sigma_{11}^{2}(t)=\frac{D_{V} t}{A^{2} \omega_{c}^{4}}=\frac{D_{V}}{G^{2}} t
$$

where Eq. (41) has been taken into account. Interestingly, this result is identical to the one obtained by omitting the second- and third-order terms in the vortex equation of 
motion (48). We thus conclude that these terms have two effects: First, they produce the oscillatory parts in Eq. (65) (note that they are naturally connected to the cycloidal vortex trajectories), and second, for small times the slope of $\sigma_{11}^{2}$ in Eq. (66), averaged over the oscillations, is larger by a factor of $3 / 2$ compared to Eq. (67).

We do not present here the other elements of the variance matrix because they contain even more terms than Eq. (65). Instead, we simply record the expression for their long time behavior, which is

$$
\begin{aligned}
\sigma_{12}^{2} & =\frac{D_{V}}{G^{2}} \frac{F_{0}^{\prime}}{2 G} t^{2}, \\
\sigma_{22}^{2} & =\frac{D_{V}}{G^{2}}\left[t+\frac{1}{3}\left(\frac{F_{0}^{\prime}}{G}\right)^{2} t^{3}\right] .
\end{aligned}
$$

The quadratic and cubic terms in $t$ appear in addition to the standard random walk result which is proportional to $t$. These additional terms arise because we have allowed that the driving force $\mathbf{F}$ depends on the vortex position, see Eq. (34). We have considered a force in the $X_{1}$ direction which drives the vortex in the $X_{2}$ direction, due to the gyrocoupling force $\mathbf{G}_{V} \times \dot{\mathbf{X}}$ in Eq. (2) or $\mathbf{G} \dot{\mathbf{X}}$ in Eq. (7), respectively. Therefore, only the 2-components of $\sigma^{2}$ are affected, $\sigma_{12}^{2}$ acquiring a factor $\left(F_{0}^{\prime} / G\right) t, \sigma_{22}$ acquiring it twice.

\section{Langevin dynamics simulations}

\subsection{Numerical procedure}

We begin with one vortex with its center located at a distance $R_{0}$ from the middle of a circularly shaped square lattice with a radius of $L$ lattice constants. We use free boundary conditions to get an image antivortex which leads to a radial force on our vortex (see [11,26] and below). The initial spin configuration stems from an iterative program which produces a discrete vortex structure on the lattice [24]. In this way we avoid the radiation of spin waves which would appear during the early time units if we use a continuum approximation for the vortex shape. The parameter ranges must be chosen very carefully for the following reasons: i) We want that the vortex moves smoothly over the Peierls-Nabarro potential of the lattice; hence, the diameter $2 r_{v}$ of the out-of-plane structure must be considerably larger than the lattice constant. Setting $\delta=0.1$ we find $2 r_{v} \simeq 3$ from Eq. (19); ii) we choose a system radius $L=24$ which provides enough space: the vortex moves outwards roughly on a spiral, but even for very long integration times the out-of-plane vortex structure should not contact the boundary, and iii) for the same reason the initial distance $R_{0}$ from the middle of the circle should not be too large. On the other hand, $R_{0}$ should not be too small; otherwise the driving force $\mathbf{F}$ would not be strong enough to overcome the pinning forces of the lattice. Letting $R_{0}=10$ both conditions can be simultaneously fulfilled, if the damping $\epsilon$ is small enough (the larger $\epsilon$, the sooner the vortex reaches the boundary). Note however that a small $\epsilon$ means long saturation times (see below).

For the time integration of the Landau-Lifshitz equation we use the discrete version of (42) where $d \mathbf{S} / d t$ has already been isolated on the l.h.s. . In contrast to our analytical calculations we work here with the cartesian components $S_{\alpha}$. Therefore we explicitly take into account the constraint $\mathbf{S}^{2}=1$ by adding $\mathbf{S}$ times a Lagrange parameter $\lambda$ to (42), see Ref. [25]. We form the time derivative of the constraint, eliminate $\lambda$ and get

$$
\frac{\mathrm{d}}{\mathrm{d} t} \mathbf{S}=\mathbf{U}+\frac{\mathbf{S U}}{S^{2}} \mathbf{S}
$$

with

$$
\mathbf{U}=\frac{1}{1+\epsilon^{2} S^{2}}\left(-\mathbf{S} \times \frac{\delta H}{\delta \mathbf{S}}+\epsilon \mathbf{S} \times\left[\mathbf{S} \times \frac{\delta H}{\delta \mathbf{S}}\right]\right)+\boldsymbol{\eta}^{\prime},
$$

where the site index has been omitted. We note that (70) is the same as orthogonalizing $\dot{\mathbf{S}}$ and $\mathbf{U}$ by the GramSchmidt method. For the time integrations we use the same code as in [11]. In addition, the position of the vortex center, in particular the position within a lattice cell, is determined by a procedure also discussed in [11].

To find a proper damping constant we checked the time dependence of the system energy using different damping constants for $L=24$ and $T=0.02$. The energy at $t=0$ is the same as for $T=0$ and $\epsilon=0$ because the noise will be introduced with the first time step of the simulation. The energy then rises and saturates on a value independent of $\epsilon$, but for $\epsilon>8 \times 10^{-3}$ the energy decreases slowly after saturation. The saturation time gets longer with lower $\epsilon$, for $\epsilon \geq 2 \times 10^{-3}$ we achieve acceptable saturation times $<300$ (in units of $\hbar /(J S)$ ). We have always made a prerun of length $t_{0}>300$ prior to beginning the evaluation of the simulation data.

The difference between the energy without temperature and the saturation energy with temperature must be the thermal energy. We computed the mean thermal energy per spin at several temperatures and it agreed with $f / 2 \times k_{\mathrm{B}} T$ up to $T=0.9, f$ being the number of degrees of freedom per spin. For higher temperatures we find too low values for the energy. We believe that the numerical procedure would have to be improved if we were interested in this regime.

\subsection{Vortex trajectory}

We studied the trajectory of the vortex center at different temperatures keeping $L=24$ and $\epsilon=2 \times 10^{-3}$ fixed. We can distinguish three temperature regimes in which the trajectories differ qualitatively.

For $0 \leq T<T_{3} \approx 0.05$ we observe two frequencies in the oscillations around the mean trajectory which can be identified with the cycloidal frequencies $\omega_{1,2}$ in (38). The intensities of the Fourier peaks at $\omega_{1,2}$ decrease with temperature and vanish at $T_{3}$ in the background, but $\omega_{1,2}$ are constant in the whole regime. This means that here 

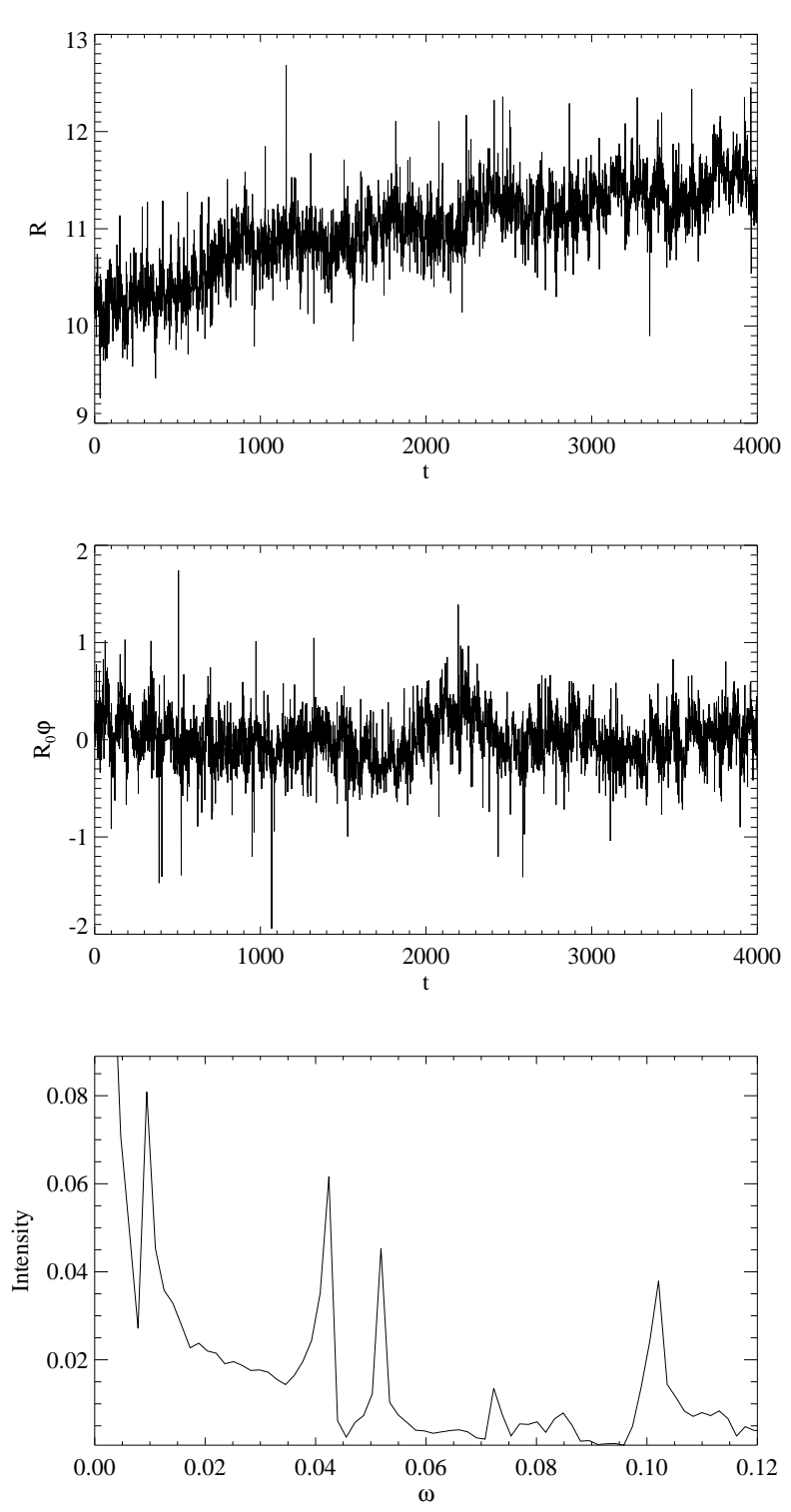

Fig. 2. Average trajectory of a vortex for temperature $T=$ 0.03 , damping $\epsilon=0.002$, system radius $L=24$ and an ensemble of 100 realizations. Upper panel: Radial coordinate of the vortex center vs time. Middle panel: Azimuthal displacement $\varphi=\phi(t)-\omega_{0} t$, where $\omega_{0}$ is the angular velocity on the mean trajectory of radius $R_{0}$. Lower panel: Fourier spectrum of $R(t)$ in the upper panel. The spectrum of $\varphi(t)$ is very similar.

the third order equation of motion (48) with temperature independent parameters can describe the vortex dynamics. For one temperature of this regime we plot in Fig. 2 the average radial coordinate $R(t)$ and the azimuthal displacement $\varphi(t)=\phi(t)-\omega_{0} t$. We want to stress that the plots present averaged results: For the computations of the vortex trajectories and variances we have averaged over 100 different runs starting from the same initial configuration, which is defined as the final configuration after a pre-run of length $t_{0}=1250$. In the expression for $\varphi(t)$ above, $\omega_{0}=F_{0} /\left(G R_{0}\right)$ is the frequency of the rotation on the mean trajectory which is essentially a circle where the radius $R_{0}$ grows very slowly with rate $g F_{0} / G^{2}$ due to the damping. On the mean trajectory the vortex is driven by a radial force $F_{0}$ due to the image vortex at $R^{(\mathrm{i})}=L^{2} / R_{0}$, which has opposite vorticity but the same polarization [26]. As the average motion is very slow $\left(\omega_{0} \approx 2.5 \times 10^{-3}\right)$ we can actually work in a cartesian system and use the results (33)-(38). Here the $X_{1}$-axis points in the radial direction, and the $X_{2}$-axis in the azimuthal direction [27]. The lowest panel of Fig. 2 shows the Fourier spectrum of $R(t)$. In addition to $\omega_{1,2}$ one also observes the difference $\Delta \omega=\omega_{2}-\omega_{1}$. This can be explained by working in polar coordinates, which is not discussed here because the formulas become much too cumbersome. The peaks at higher frequencies are second harmonics of $\omega_{1,2}$.

For $T_{3}<T<T_{1} \approx 0.3$ we do not observe the above mentioned two frequencies any longer. In this regime, we found that some runs had to be excluded from the average because the vortex suddenly changed its direction of motion. This occurs because, opposite to the case of the vorticity $q$, the polarization $p$ of the vortex is not a constant of motion for a discrete system: The out-of-plane vortex structure can flip to the other side of the lattice plane due to the stochastic forces acting on the spins. Then $G=2 \pi q p$ in Eq. (27) changes sign and thus the direction of the gyrovector in Eq. (2) is reversed, which implies that the direction of the vortex motion is reversed as well. This noise-induced switching between the two vortex polarizations is a very novel effect in itself, and hence we are developing a theory for the switching rate [28]. In this respect, it can be mentioned that switching can also be induced by an ac magnetic field in the easy plane. As the symmetry is broken here, such a switching occurs only for one sense of rotation, and there is no transition back to the original state [29].

Finally, for $T>T_{1}$, a single-vortex theory as presented here is no longer adequate because at these temperatures the probability for the spontaneous appearance of vortexantivortex pairs becomes too large. These pairs can break up above the Kosterlitz-Thouless transition temperature $T_{K T} \simeq 0.7$ in our units. Between $T_{1}$ and $T_{K T}$, these pairs interact with the initial vortex although they are not separated, thus introducing new forces and effects which the present theory does not take into account. Moreover, very recent Monte Carlo simulations [30, 31, 32] have revealed that for higher temperatures the vortex motion is strongly influenced by creation and annihilation processes: Typically, an unbound vortex travels only one or a few lattice spacings until it annihilates with the antivortex of a pair which meanwhile appeared spontaneously in the neighborhood. Then, the vortex of this pair continues the travel instead of the original vortex.

\subsection{Variances of the vortex trajectories}

As the vortex positions in the simulations are evaluated in polar coordinates, we obtain a variance matrix with elements $\sigma_{R R}^{2}, \sigma_{R \phi}^{2}$ and $\sigma_{\phi \phi}^{2}$. Their time evolution is plotted in Fig. 3 for $T=0.03$, which is close to the upper 

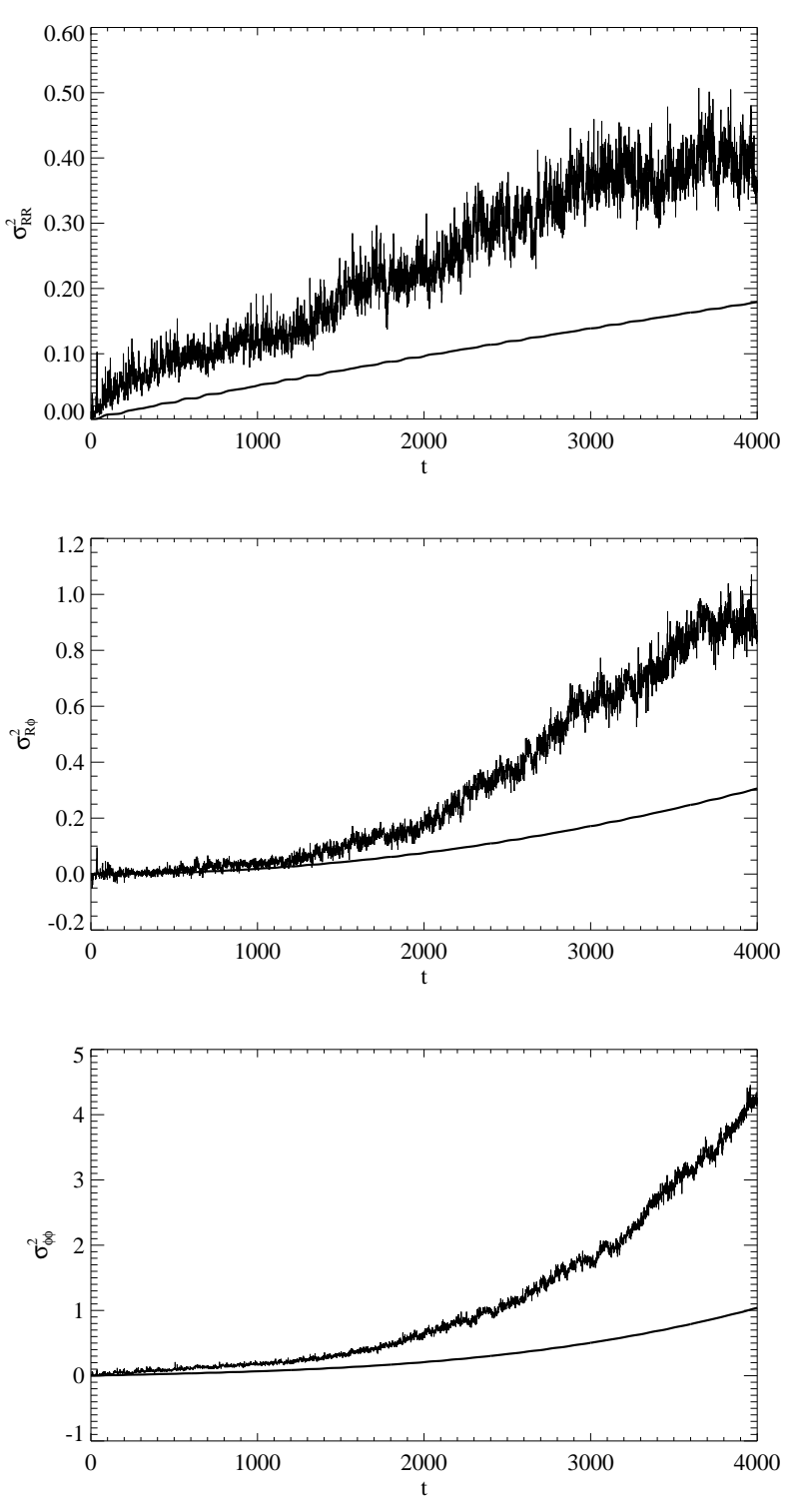

Fig. 3. Variances of the vortex trajectory; parameters are the same of Fig. 2. From top to bottom, shown are the variance of the radial coordinate, $\sigma_{R R}^{2}=\left\langle R^{2}\right\rangle-\langle R\rangle^{2}, \sigma_{R \phi}^{2}$, the off-diagonal elements of the variance matrix, and $\sigma_{\phi \phi}^{2}=$ $\left\langle\left(R_{0} \varphi\right)^{2}\right\rangle-\left\langle\left(R_{0} \varphi\right)\right\rangle^{2}$. In all three cases the lower line is the theoretical prediction without adjustment of parameters.

edge of the low temperature regime defined in the previous subsection. The solid lines are the theoretical results from Section 3, without the simplifications i)-iv) discussed above Eq. (65), which were only made there to facilitate the discussion. As the theory has been worked out in cartesian coordinates, the following factors appear when going over to polar coordinates: No factor in $\sigma_{R R}^{2}$, a factor $\kappa=1-F_{0} /\left(F_{0}^{\prime} R_{0}\right)$ in $\sigma_{R \phi}^{2}$, and a factor $\kappa^{2}$ in front of the terms cubic in time in $\sigma_{\phi \phi}^{2}$.

Figure 3 shows that, for not too long times, the agreement between theory and simulation is astonishingly good; it is important to stress that no parameters were adjusted at all. Moreover, we worked in the continuum limit, while the simulations were performed on a discrete system. For very long times, $t \geq 2000$, the agreement becomes poorer. This is partially due to one simplification of the theory, namely that we have used a constant $R_{0}$ although during the simulation $R_{0}$ slowly increases by several lattice constants as the trajectory is roughly a spiral (see the cartoon in Fig. 1). The force term $F_{0}^{\prime}=F^{\prime}\left(R_{0}\right)$ increases as well, because the force increases when the distance to the image vortex becomes smaller. As $F_{0}^{\prime}$ appears in $\sigma_{R \phi}^{2}$ and $\left(F_{0}^{\prime}\right)^{2}$ arises in front of the cubic term in $\sigma_{\phi \phi}^{2}$ in Eqs. (68) and (69), including this effect would lead to an improvement of the agreement between theory and simulations.

Aside from those discussed above, there is another possible reason for the discrepancy between theory and simulation whose consideration, unfortunately, would lead to very involved calculations: The integral (52) for $D_{V}$ [as well as the integrals in Sec. 22 except (9)] have been evaluated by placing the vortex into the middle of the circular system. However, in the simulations the distance from the lattice center is $R_{0}$, which moreover increases slowly. We have estimated the above integrals by expanding in $R_{0} / L$, which shows that the first order terms vanish. Nevertheless, the second order terms yield corrections which are already of the order of $20 \%$ for $R_{0}=10$, becoming larger as $R_{0}$ increases. Even more, the variance (54) of the stochastic forces is actually a diagonal tensor, see Eq. (51) and [21. Therefore, we get a radial diffusion constant $D_{V}^{(R)}$ which differs from the azimuthal constant $D_{V}^{(\phi)}$ when the vortex is not at the center. This splitting is also of order $\left(R_{0} / L\right)^{2}$. As $D_{V}^{(\phi)}$ appears, e. g., in front of the cubic term in $\sigma_{\phi \phi}^{2}$, whereas the linear term contains $D_{V}^{(R)}$, it is quite possible that the agreement with the simulations could be improved by taking into account the splitting of the diagonal elements of the diffusion tensor.

We numerically integrated up to times $t=4000$ (let us point out in this regard that this takes three weeks CPU time on a CRAY-YMP/EL for averages over 100 runs) because this is the characteristic time given by $1 / \beta_{c}=8 / \epsilon$ for the damping in the trajectories. We should see then that the slope of the time-averaged function $\sigma_{R R}^{2}$ gradually decreases, eventually by a factor one third for $t \gg 1 / \beta_{c}$ [cf. the discussion of Eq. (65)]. We checked this for the theoretical results in Fig. 3, and found that in the simulation data this effect can be observed only qualitatively. For $\sigma_{R \phi}^{2}$ and $\sigma_{\phi \phi}^{2}$ the effect is hidden by the quadratic and cubic terms.

We would like to stress that the strong fluctuations in Fig. 3 (which seem to be smaller in the two lower plots because of the different scales) arise not only due to the noise but also from discreteness effects. This is demonstrated very clearly by the simulation in Fig. 1 for a very low temperature $(T=0.003)$ using 1500 realizations. We have identified the sharp spikes as discreteness effects by comparing with the times when the vortex center moves over the ridges along the lattice lines (these times are indicated as dashed vertical lines). The vortex energy is high- 


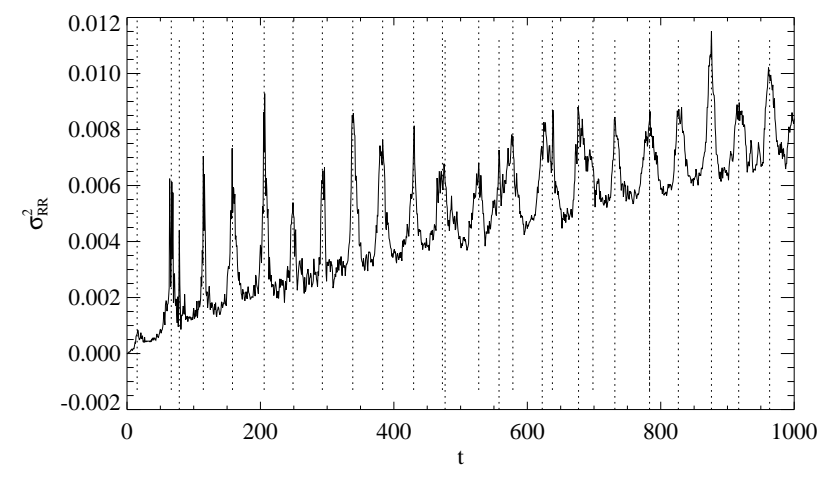

Fig. 4. Variance of the radial vortex coordinate averaged over 1500 realizations, for $T=0.003, \epsilon=0.002$ and $L=24$. The dashed vertical lines indicate the times at which the vortex center moves over ridges of the Peierls-Nabarro periodic potential.

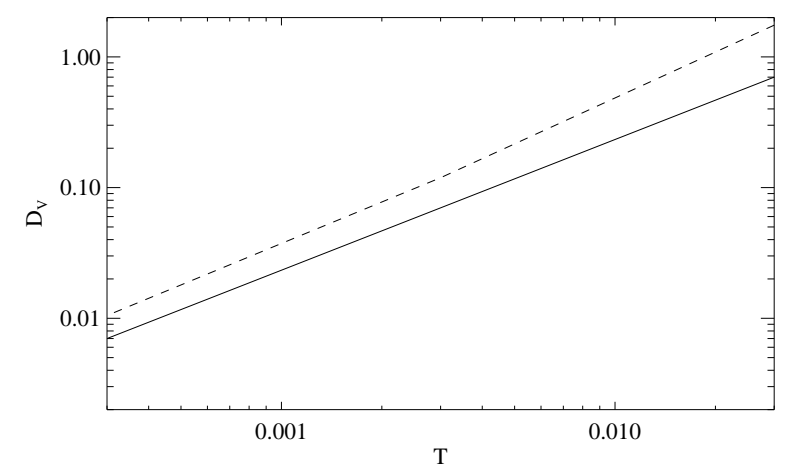

Fig. 5. Vortex diffusion constant $D_{V}$ as a function of temperature, for $\epsilon=0.002$ and $L=24$. Solid line: Theoretical results from Eq. (53); dashed line: Adjusted $D_{V}$ from fitting the theoretical curves for $\sigma^{2}(t)$ to the simulation data.

est when the center is at a lattice point, and lowest in the middle of a cell.

Last, but not least, we discuss the temperature dependence of the vortex diffusion constant $D_{V}$. A linear dependence is predicted by Eqs. (53) and (54). For comparison with the simulations we have fitted the theoretical curves to the observed variances by adjusting $D_{V}$, which appears as a factor in front of all the components of $\sigma^{2}$. This was done for two temperature decades. Fig. 5 shows a nearly linear dependence, and therefore the only difference between $D_{V}$ from the simulations and the theoretical $D_{V}$ is a constant factor of about 1.8 for the whole temperature regime.

\section{Conclusions}

In this paper, we have reported our analytical and numerical work regarding the effects of temperature on the dynamics of non-planar vortices in 2D, classical, anisotropic Heisenberg ferromagnets. As a preliminary result, we have described the zero temperature dynamics of vortices in the presence of Gilbert damping. We found that damping contributes to all the terms of the third order equation of motion for the vortex position, but its contribution is always an order smaller in the system size than the corresponding free propagation part. We have solved the equations of motion and qualitatively discussed the motion of the vortex, which consists of a mean straight trajectory plus (damped) additional oscillations. By means of the same analytical approach, we have been able to derive a third order stochastic equation of motion for the vortex center when thermal noise is added to the system. The equation shows that the effective stochastic force acting on the vortex is also a Gaussian white noise, whose variance depends linearly on the temperature. We have exactly solved the stochastic equation of motion and obtained analytical expressions for the mean vortex trajectory and its variance. The variance along the coordinate perpendicular to the direction of motion of the vortex is diffusive, i.e., it increases linearly with time; however, other components of the variance matrix (the parallel-perpendicular and the parallel-parallel terms) turn out to include nonlinear contributions coming from the fact that the vortex motion is perpendicular to the driving force, due to a Lorentz-like gyrocoupling force.

The above summarized analytical results, obtained in the continuum limit of the Landau-Lifshitz equations governing the model dynamics, have been compared to Langevin dynamic simulations of the discrete 2D Heisenberg model. The numerical results allow us to establish three different temperature regimes for the vortex propagation: a low temperature one, where the vortex motion follows essentially the third order equation of motion with parameters independent of temperature; a middle temperature one, at which traces of the oscillations arising from the third order equation are lost, and a high temperature regime, which is not describable by a one-vortex approach because too many vortex-antivortex pairs arise in the system. Our analytical results are seen to be a good description of the vortex dynamics up to temperatures of the order of $10 \%$ of the Kosterlitz-Thouless transition temperature. Remarkably, the analytical predictions, which include no adjustable parameters, agree qualitatively well with the numerical simulations, and even quantitatively at early times. The agreement becomes worse for longer times due to the approximations involved in our theory: The calculations were made for a constant radius of the trajectory and a constant force $\mathbf{F}$ gradient, aside from simplifications necessary to calculate the integrals which give the parameters for the equation of motion. In addition, we have been able to clearly identify the influence of discreteness in the numerical results, which cannot be captured by our continuum theory. Finally, we have also verified that the vortex diffusion constant depends linearly on temperature as predicted, although the quantitative comparison is not correct by a factor two. We thus conclude that the collective coordinate theory we have derived for vortex dynamics is a good description of the phenomena observed numerically at low and intermediate temperatures. The 
discrepancies between theory and simulations have been understood in terms of the unavoidable approximations involved in the calculations. Finally, we note that for vortices quantum effects are possibly more important than for kinks in one-dimensional spin models, where at least a part of these effects can be taken into account by a renormalization of the kink parameters. For 2D spin models, it is not clear how a quantum vortex should be defined. In any case, a finite lifetime and other novel features seem to appear 33.

We thank Esteban Moro and Grant Lythe for discussions. Travel between Bayreuth and Madrid is supported by "Acciones Integradas Hispano-Alemanas", a joint program of DAAD (Az. 314-AI) and DGES. Travel between Europe and Los Alamos is supported by NATO grant CRG 971090. Work at Madrid and Leganés is supported by CICyT (Spain) grant MAT95-0325 and by DGES (Spain) grant PB96-0119. Work at Los Alamos is supported by the United States Department of Energy.

\section{References}

1. Nonlinearity in Condensed Matter, edited by A. R. Bishop, R. Ecke, and J. Gubernatis (Springer, Berlin, 1993).

2. Nonlinear Coherent Structures in Physics and Biology, edited by K. H. Spatschek and F. G. Mertens (Plenum, New York, 1994).

3. Fluctuation Phenomena: Disorder and Nonlinearity, edited by A. R. Bishop, S. Jiménez, and L. Vázquez (World Scientific, Singapore, 1995).

4. See, e.g., A. R. Bishop, in Ref. [3

5. A. Sánchez and A. R. Bishop, SIAM Review in press (1998).

6. A. R. Völkel, F. G. Mertens, A. R. Bishop and G. M. Wysin, Phys. Rev. B43, 5992 (1991).

7. M. E. Gouvea, G. M. Wysin, A.R. Bishop and F. G. Mertens, Phys. Rev. B39, 11840 (1989).

8. G. M. Wysin, Phys. Lett. A, in press.

9. A. A. Thiele, Phys. Rev. Lett. 30, 230 (1973).

10. A. A. Thiele, J. Appl. Phys. 45, 377 (1974).

11. F. G. Mertens, H.-J.Schnitzer and A.R. Bishop, Phys. Rev. B 56, 2510 (1997).

12. G. M. Wysin, F. G. Mertens, A. R. Völkel and A. R. Bishop, in [2].

13. A. R. Völkel, G. M. Wysin, F. G. Mertens, A. R. Bishop, and H. J. Schnitzer, Phys. Rev. B 50, 12711 (1994).

14. F. G. Mertens, A. R. Bishop, G. M. Wysin, and C. Kawabata, Phys. Rev. B 39, 591 (1989).

15. J. F. Currie, J. A. Krumhansl, A. R. Bishop and S. E. Trullinger, Phys. Rev. B 22, 477 (1980).

16. K. Hirakawa, H. Yoshizawa, J. D. Axe, and G. Shirane, Suppl. J. Phys. Soc. Jpn. 52, 19 (1983); L. P. Regnault, J. P. Boucher, J. Rossat-Mignod, J. Bouillot, R. Pynn, J. Y. Henry, and J. P. Renard, Physica B+C 136B, 329 (1986); M. T. Hutchings, P. Day, E. Janke, and R. Pynn, J. Magn. Magn. Mater. 54-57, 673 (1986); S. T. Bramwell, M. T. Hutchings J. Norman, R. Pynn, and P. Day, J. de Phys. 49, C8-1435 (1988); D. G. Wiesler, H. Zabel, and S. M. Shapiro, Physica B 156-7, 292 (1989); D. G. Wiesler, H. Zabel, and S. M. Shapiro, Z. Physik B 93, 277 (1994);
L. P. Regnault, C. Lartigue, J. F. Legrand, B. Farago, J. Rossat-Mignod, and J. Y. Henry, Physica B 156-7, 298 (1989).

17. P. Gaveau, J. P. Boucher, L. P. Regnault, and Y. Henry, J. Appl. Phys. 69, 6228 (1991).

18. D. L. Huber, Phys. Rev. B26, 3758 (1982).

19. The sign of our damping term differs from [9, 10,20] because we work with spins while these authors deal with magnetisations.

20. S. Iida, J. Phys. Chem. Solids 24, 625 (1963).

21. T. Kamppeter, F. G. Mertens, A. Sánchez, N. GrønbechJensen, A. R. Bishop, and F. Domínguez-Adame, in "Theory of Spin Lattices and Lattice Gauge Models," eds. M. L. Ristig, K. A. Gernoth and J. W. Clark. Springer-Verlag, Berlin (1997).

22. The correlation between different components of $\mathbf{F}^{\text {st }}$ can also be calculated and turns out to be zero.

23. S. Miyashita, H. Nishimori, A. Kuroda and M.Suzuki, Progress of Theor. Phys. 60, 1669 (1978).

24. H.-J.Schnitzer, Zur Dynamik kollektiver Anregungen in Hamiltonschen Systemen, Ph.D.-thesis, University of Bayreuth (1996).

25. N. Grønbech-Jensen and S. Doniach, J. Comp. Chem. 15, 997 (1994).

26. F. G. Mertens, G. M. Wysin, A. R. Völkel and A. R. Bishop, in [2].

27. Strictly speaking, Eq. (48) must be solved in polar coordinates which leads to shifts of $\omega_{1,2}$ by $\pm \omega_{0}$, respectively 11. The different signs appear due to the phase differences $\pm \pi / 2$, below Eq. (38).

28. Yu. Gaididei, T. Kamppeter, F. G. Mertens and A. R. Bishop, submitted (August 1998).

29. Yu. Gaididei, T. Kamppeter, F. G. Mertens and A. R. Bishop, in preparation.

30. D. A. Dimitrov and G. M. Wysin, Phys. Rev. B 53, 8539 (1996).

31. J. E. R. Costa, B. V. Costa, and D. P. Landau, Phys. Rev. B 57, 11510 (1998).

32. D. A. Dimitrov and G. M. Wysin, preprint (March 1998).

33. J. Schliemann, F. G. Mertens, and H. Frahn, in preparation. 